\title{
Information transport by sine-Gordon solitons in microtubules
}

\author{
Elcio Abdalla ${ }^{1}$, Bouchra Maroufi ${ }^{1,2}$, Bertha Cuadros Melgar ${ }^{1}$ and Moulay Brahim Sedra ${ }^{2,3}$ \\ ${ }^{1}$ Instituto de Fúsica, Universidade de São Paulo \\ C.P.66.318, CEP 05315-970, São Paulo, Brazil \\ ${ }^{2}$ Laboratoire-UFR, de Physique des Hautes Energies, Faculté des Sciences, \\ Av Ibn Batouta, BP 1014, Rabat, Morocco. \\ ${ }^{3}$ Laboratoire de Physique Théorique et Appliquée (L.P.T.A), Départment de Physique \\ Faculté des Sciences, BP 133, Kénitra, Morocco
}

$(01 / 12 / 01)$

\begin{abstract}
We study the problem of information propagation in brain microtubules. After considering the propagation of electromagnetic waves in a fluid of permanent electric dipoles, the problem reduces to the sine-Gordon wave equation in one space and one time dimensions. The problem of propagation of information is thus set.
\end{abstract}

PACS number(s): 87.17.Aa 87.17.-d 89.70.+c 89.75.Fb 89.75.-k

Information is a central question in the understanding of the mechanisms regulating the brain. Questions as bounds on information, capacity of communication channels are of extreme relevance and a theory of information and communication is of utter value [1]. Thus, how much information can be stored by a cube is an important question. Foreseeable technology making use of atomic manipulation would suggest an upper bound of around $10^{20}$ bits. But as technology takes advantage of unforeseen paradigms, this number could grow up. Could the bound grow without limit? With black hole thermodynamics some definite answers are forthcoming [2]. In Quantum Field Theory the question developed unexpectedly in the framework of black hole thermodynamics and Quantum Gravity [3]. Some knowledge was already known from Shannon's Theory of Information as well [ 4 .

Shannon imagined a system capable of storing information by virtue of it possessing many distinguishable states. A state $a$ is not known a priori but its probability $p_{a}$ is known. The measure of uncertainty corresponds to an entropy $S$ which formally coincides with the corresponding statistical interpretation of entropy:

$$
S=-K \sum_{a} p_{a} \ln p_{a}
$$

On the other hand, it has been conjectured [5] that the brain microtubules might be an active component of the brain functioning. It is thus natural to consider electromagnetic waves moving in that cavity as transporting and carrying information.

With these matters in mind, we consider here the effective electromagnetic wave obtained when the second quantized electromagnetic field interacts with the permanent dipole moment of the vicinal water in brain microtubules. The second quantized electromagnetic field shall be given by the usual development in frequency components [6]. The electric dipoles can be seen as two-component spinors described as effective spin-fields. Therefore, we have, for the effective Hamiltonian,

$$
H=H_{Q E D}-\mu \sum_{j=1}^{N}\left[\vec{E}_{t r}\left(\vec{x}_{j}, t\right) \vec{s}\right]+\epsilon \sum_{j=1}^{N} s_{z},
$$

where $\vec{E}_{t r}$ is the electric field transversal to the wave propagation direction and $\vec{s}$ is a spin field describing the electric dipole moment degree of freedom. The last term represents an effective interaction of the $z$-component of the electric dipole with an average electric field, that is, it represents a two-energy eigenstates system. The value of $\epsilon \approx 50 \mathrm{~cm}^{-1}=6.3 \times 10^{-3} \mathrm{eV}=1.0 \times 10^{-14} \mathrm{erg}$ has been claimed in [7].

Such a problem has been considered by [8]. We derive some results which have not been explicitely obtained in [8]. We suppose that the electromagnetic field has a fast dependence on $z-c t$ and a slow dependence on $z$ and $t$, allowing us to write the expansion

$$
\vec{E}(\vec{x}, t)=\sum \vec{E}_{t r}^{n}(z, t) e^{i k_{n}(z-c t)}
$$

Using the equations of motion derived from the Hamiltonian (2), that is, Maxwell equations with sources, we arrive at

$$
\frac{\partial E^{ \pm}}{\partial z}+\frac{1}{c} \frac{\partial E^{ \pm}}{\partial t}= \pm i \frac{2 \pi \epsilon \mu}{\hbar V} s^{\mp}
$$

This is a quantum equation of motion. However we do not have any practical means to either measure the variable $s$, or take care of its detailed dynamics, therefore we take its quantum average. Such an average is easily obtained due to the simple description of $s$ in terms of Pauli matrices, leading to a result written in terms of the exponential of the field $\theta$, defined by

$$
\theta^{ \pm}(z, t)=\frac{\mu}{\hbar} \int_{0}^{t}\left\langle E^{ \pm}(z, t)\right\rangle_{q u} d u
$$

where we take the quantum average \langle\rangle$_{q u}$. Following uch a procedure in equation (4) leads to the semiclassical equation of motion 


$$
\frac{\partial^{2} \theta^{ \pm}}{\partial t \partial \sigma}=-\frac{4 \pi \epsilon N \mu^{2}}{\hbar^{2} V} \sin \theta^{ \pm}
$$

where $N / V$ is the number of dipoles (molecules) per unit volume, and $\sigma=t+\frac{z}{c}$. Above, the indices \pm correspond to the usual combinations of the transversal direction, and we supposed also that the longitudinal direction does not propagate. This is a variant of the well known sineGordon equation. The one-soliton solution is given by the expression

$$
E=\frac{\hbar}{\mu} A \operatorname{sech} A\left(t-\frac{z}{\nu_{0}}\right)
$$

where the angular frequency characteristic of the model is

$$
A=\sqrt{\frac{2 \pi \epsilon \mu^{2} N \nu_{0}}{\hbar^{2} V\left(c-\nu_{0}\right)}}
$$

and $\nu_{0}$ is the velocity of the soliton.

In order to understand the propagation of information in such a device, we follow [1] and consider small perturbations around the soliton, which amounts to solving the equation

$$
\omega^{2} \eta+i \omega \frac{\partial \eta}{\partial t}-A_{0}^{2} \cos \theta_{0} \eta=0
$$

where $A_{0}=\sqrt{2 \pi \epsilon \frac{N}{V}} \frac{\mu}{\hbar} \approx 3.1 \times 10^{14} \mathrm{~s}^{-1}$. Plugging in back the solution $\theta_{0}=4 \arctan \exp \left[-A z / \nu_{0}\right]$, that is, $\cos \theta_{0}=$ $1-2 \operatorname{sech}^{2}\left(A z / \nu_{0}\right)$, we obtain the equation

$$
i \omega \frac{\partial \eta}{\partial t}=-2 A_{0}^{2} \operatorname{sech}^{2}\left(A z / \nu_{0}\right) \eta
$$

where we chose the boundary conditions such that $\omega=$ $A_{0}$. The only solution is

$$
\eta=\exp \left[2 i \sqrt{\frac{\nu_{0}}{c}} \tanh \frac{A z}{\nu_{0}}\right]
$$

Let us discuss the physics behind the problem and the consequences for the constants appearing in the solution. First, the constant $\epsilon$ is a free parameter and represents the energy of a dipole in the vicinal water. It is of the order of magnitude of difference of two molecular energy levels. The study of vibration in water indicates the value $\epsilon \approx 50 \mathrm{~cm}^{-1}=6.3 \times 10^{-3} \mathrm{eV}=1.0 \times 10^{-14} \mathrm{erg}$ [7]. The constant $\mu$ represents the permanent electric dipole moment of the water, which is the electron charge times $0.2 \times 10^{-8} \mathrm{~cm}$, that is, in CGS units, $\mu \approx 6.8 \times 10^{-18}$. Finally, the number of molecules per unit volume is easily obtained for the water, it is of the order of $0.3 \times 10^{23} \mathrm{~cm}^{-3}$.

In order to fix the velocity of the wave, we integrate the solitonic electric field imposing that it is the unit synaptic potential coming from the quantum of transmitter producing a postsynaptic potential, typically 0.5 to $1.0 \mathrm{mV}$, as discussed in [9], who has proposed it to be a quantum unit of potential in such a context. We have

$$
V \approx \int E d z=\frac{\pi}{2} \frac{\hbar \nu_{0}}{\mu},
$$

thus obtaining, for the velocity parameter, the value $\nu_{0} \approx$ $1.4 \times 10^{4} \mathrm{~cm} / \mathrm{s}$, or $\frac{\nu_{0}}{c} \approx 0.5 \times 10^{-6}$. With this result for $\nu_{0}$ we obtain for the constant $A$ the result $A \approx 2.2 \times$ $10^{11} \mathrm{~s}^{-1}$. Estimating the time to send information as that necessary to pass the bulk of the soliton (7), we get a rough estimate for the frequency of the waves as $\nu \approx$ $\frac{A}{6} \approx 3.7 \times 10^{10} \mathrm{~s}^{-1}$.

On the other hand, taking the average electric field in the brain as corresponding to the quantum unit of electric potential value as discussed above, namely $\sim 1 \mathrm{mV}$, divided by the lenght of the typical microtubule, that is, $\sim 10 \mathrm{~nm}$ we are led, for the average electric field, to the value

$$
E_{\text {ave }} \approx 10^{-1} V 2 \times 10^{2} \times 10^{-9} \mathrm{~m} \approx 3 \text { stat } \mathrm{C} / \mathrm{cm}
$$

Now, the constants $A$ and $E_{\text {ave }}$ are related by

$$
A=E_{\max } \frac{\mu}{\hbar}=\sqrt{2} E_{\text {ave }} \frac{\mu}{\hbar} \approx 4 \times 10^{10} s^{-1}
$$

This is compatible with the previous value for $A$, giving us some confidence on the result.

The corresponding wavelenght is $\lambda=\frac{c}{2 \pi A} \approx 3 \mathrm{~mm}$. This corresponds to the order of magnitude of the pineal gland. Whether this is just a coincidence or whether it has a deeper meaning is a question that deserves further study. Moreover, it corresponds to a typical frequency already obtained for phonon transitions in the brain, allowing for new theoretical models of the interaction of electromagnetism with the biological cells [10]!

Furthermore, there are bounds on information storage. Theoretically, in a problem of completely different character, one arrives at the maximum entropy a cache can hold, with the result

$$
I_{\text {max }}<\frac{2 \pi R \mathcal{E}}{\hbar c \ln 2}
$$

where $R$ is the overall radius of the object under study and $\mathcal{E}$ its energy.

In the theory of solitons, the number of possible information-holding configurations based on the soliton equals the number of quanta that might populate the first excited level. To this number we must add unity to account for the soliton configuration itself. So, the possible configurations within an energy budget $\mathcal{E}$ above the soliton energy is $N(\mathcal{E})=1+[[\mathcal{E} / \hbar \omega]]$ ([[x]] stands for the integral part of $x$ ), then

$$
I_{\text {max }}=\ln \left(1+\left[\left[\mathcal{E} / \omega_{1}\right]\right]\right) \log _{2} e \text { bits }
$$

consistent with the bound (15). In our case, for a microtubule, we have $R \approx 10^{-4} \mathrm{~cm}$. Taking the energy $\mathcal{E}$ as corresponding to a quantum of energy $\hbar A_{0}$, from the source 
of the electromagnetic field, we find $\mathcal{E}=\hbar A_{0} \approx 0.2 \mathrm{eV}$. In such a case,

$$
I_{\max } \approx 1
$$

while the bound (15) corresponds to $I_{\max }<6$.

It would not be too original to call such an information a quantum information unit sent via the microtubuiles, in view of similar considerations, in a diferent context by Gabor [11. In the present case, the soliton, formed by the interaction of Quantum Electrodynamics with the electric dipole moment of the background water in the onedimensional device offered by microtubules is the natural way chosen by nature to send information bits.

Another interesting point is the fact that the frequency parameters which showed up naturally in the course of the computations have natural interpretations in terms of brain structures. The frequency $A_{0} \approx 3.1 \times 10^{14} \mathrm{~s}^{-1}$ is compatible with the size of the microtubules. But the real frequency $\nu \approx \frac{A_{0}}{6} \sqrt{\frac{\nu_{0}}{c}} \approx 3.7 \times 10^{10} \mathrm{~s}^{-1}$ is compatible with the transition period observed for the socalled conformational changes connected with tubulin dimer protein (namely $\approx 10^{9}$ to $10^{11} \mathrm{~s}^{-1}$ ) [8].

This is a further example of the application of Quantum Field Theory to general aspects of matter interactions in complex systems. It is clear that this is not a way of achieving comprehension of the complexity aspects of such a sophisticated system, but it certainly provides valuable tools for working in this field as well.

Acknowledgements: this work has been partially supported by CNPq (Conselho Nacional de Desenvolvimento Científico e Tecnológico) and FAPESP (Fundação de Amparo à Pesquisa do Estado de São Paulo). B.Maroufi would like to thank the Instituto de Física, Universidade de São Paulo, for the hospitality. E.A. thanks Drs. I. Prates de Oliveira and S. F. de Oliveira for discussions concerning aspects of the brain functions.

[1] J. Bekenstein and M. Schiffer Int. J. Mod. Phys. C 1 (1990) 355-422.

[2] J.D.Bekenstein, Lett. Nuov. Cim. 4 (1972) 737; Phys. Rev. D7 (1973) 2333; D9 (1974) 3292; S. Hawking, Commun. Math. Phys. 43 (1975) 199.

[3] G. 't Hooft, gr-qc/9310026; L. Susskind, J. Math. Phys. 36 (1995) 6377.

[4] C. Shannon and W. Weaver, The Mathematical Theory of Communication, Univ. of Illinois Press, 1949.

[5] R. Penrose The Emperor's New Mind, Oxford University Press, 1989, Shadows of the Mind, Oxford University Press, 1994.

[6] C. Itsykson and J.B. Zuber Quantum Field Theory, Mcgraw-hill, New York, (1980).
[7] F. Franks Water: A comprehensive Treatise, Plenum Press, New York, 1972.

[8] M. Jibu S. Hagan, S. R. Hameroff, K. H. Pribram and K. Yasue, Bio Systems 32 (1994) 195-209.

[9] E. R. Kandel in Essentials of Neural Science and Behavior, ed. E. R. Kandel, J. H. Schwartz and T. M. Jessel, Prentice Hall International, INC, 1995.

[10] S. R. Hameroff and R. C. Watt J. Theor. Biol. 98 (1982) 549; E. Del Giudice, G. Preparata and G. Vitiello Phys. Rev. Lett. 61 (1988) 1085.

[11] D.Gabor Nature 161 (1948) 777-778. 